\title{
Impact of Stress and Glucocorticoids on Schema-Based Learning
}

\author{
Lisa Marieke Kluen', Patricia Nixon', Agorastos Agorastos², Klaus Wiedemann² and Lars Schwabe*,I \\ 'Department of Cognitive Psychology, University of Hamburg, Hamburg, Germany; ${ }^{2}$ Department of Psychiatry, University Clinic Hamburg- \\ Eppendorf, Hamburg, Germany
}

\begin{abstract}
Pre-existing knowledge, a 'schema', facilitates the encoding, consolidation, and retrieval of schema-relevant information. Such schema-based memory is key to every form of education and provides intriguing insights into the integration of new information and prior knowledge. Stress is known to have a critical impact on memory processes, mainly through the action of glucocorticoids and catecholamines. However, whether stress and these major stress mediators affect schema-based learning is completely unknown. To address this question, we performed two experiments, in which participants acquired a schema on day I and learned schema-related as well as schema-unrelated information on day 2. In the first experiment, participants underwent a stress or control manipulation either immediately or about 25 min before schema-based memory testing. The second experiment tested whether glucocorticoid and/or noradrenergic activation is sufficient to modulate schema-based memory. To this end, participants received orally a placebo, hydrocortisone, the $\alpha 2$-adrenoceptor-antagonist yohimbine, leading to increased noradrenergic stimulation, or both drugs, before completing the schema-based memory test. Our data indicate that stress, irrespective of the exact timing of the stress exposure, impaired schema-based learning, while leaving learning of schema-unrelated information intact. A very similar effect was obtained after hydrocortisone, but not yohimbine, administration. These data show that stress disrupts participants' ability to benefit from prior knowledge during learning and that glucocorticoid activation is sufficient to produce this effect. Our findings provide novel insights into the impact of stress and stress hormones on the dynamics of human memory and have important practical implications, specifically for educational contexts.

Neuropsychopharmacology (2017) 42, |254-|26|; doi:I0.1038/npp.2016.256; published online I4 December 2016
\end{abstract}

\section{INTRODUCTION}

Prior knowledge, referred to as a schema, provides a framework for the organization and efficient incorporation of new information. Although the powerful impact of preexisting schemas on learning and memory has long been observed (Alba and Hasher, 1983; Anderson, 1984; Barlett, 1932), the neural underpinnings of schema-based memory have been elucidated only over the past decade. Rodent studies and neuroimaging studies in humans identified the medial prefrontal cortex (mPFC) as a key region in schemabased memory (Ghosh et al, 2014b; Tse et al, 2007; Tse et al, 2011; van Kesteren et al, 2010). In particular, the mPFC is thought to detect the schema-congruence of information and to facilitate the integration of schema-relevant information into the neocortical schema representation (van Kesteren et al, 2012). The hippocampus, in turn, although being critical for learning novel information, is thought to be less relevant for schema-based memory (Tse et al, 2007; van Kesteren et al, 2012).

* Correspondence: Professor L Schwabe, Department of Cognitive Psychology, University of Hamburg, Von-Melle-Park 5, Hamburg 20 I 46, Germany, Tel: +49 40 43838-5950, Fax: +49 40428384729 , E-mail: lars.schwabe@uni-hamburg.de

Received 5 August 2016; revised 7 November 2016; accepted 8 November 2016; accepted article preview online 14 November 2016
Stress is well-known to have a critical influence on learning and memory (Diamond et al, 2007; Roozendaal et al, 2009; Schwabe et al, 2012a) and the PFC is among the brain areas that are most sensitive to stress. For instance, stress may affect the goal-directed control of instrumental learning (Schwabe and Wolf, 2009), known to rely on the mPFC (Valentin et al, 2007), as well as dendritic morphology in the mPFC (Izquierdo et al, 2006; Moench et al, 2016). These effects of stress on prefrontal functions are critically mediated by glucocorticoids and noradrenaline (Barsegyan et al, 2010; Schwabe et al, 2012b). Glucocorticoid and noradrenaline effects are not necessarily independent of one another but there is compelling evidence that glucocorticoids and noradrenaline interact to decrease PFC-dependent functions (Barsegyan et al, 2010; Schwabe et al, 2011; Schwabe et al, 2010a). Although the effects of stress, glucocorticoids and noradrenaline on memory are well-established, the fundamental idea that learning often occurs against the background of existing prior knowledge has been barely considered and whether stress and major stress mediators affect schemabased memory processes is unknown.

Here, we examined the impact of stress, glucocorticoids, and noradrenaline on the use of prior knowledge during learning. We performed two experiments, in which participants acquired a schema on day 1 and learned schema-related and -unrelated material on day 2. The first experiment 
assessed the impact of stress on schema-based memory. To address potential differences in the role of noradrenaline and glucocorticoids in the putative stress effect, we employed the different temporal profiles of action of the two stress response systems. Specifically, participants performed the test of schema-based memory either immediately after the stress (or control) manipulation when noradrenergic arousal was high and cortisol levels were hypothesized to not be elevated yet, or $25 \mathrm{~min}$ thereafter when cortisol levels peaked and noradrenergic activation had returned to baseline. In the second experiment, we used a pharmacological manipulation of the glucocorticoid and noradrenergic systems to test whether glucocorticoid and/or noradrenergic stimulation is sufficient to affect schema-based learning. Therefore, participants received a placebo, hydrocortisone, the $\alpha 2$-adrenoceptor antagonist yohimbine that leads to increased noradrenergic stimulation, or both drugs before the test session on day 2. In both experiments, participants performed schema-basedlearning as well as inference trials after the stress or pharmacological manipulation. On the basis of findings indicating that stress interferes with prefrontal functions (Barsegyan et al, 2010; Elzinga and Roelofs, 2005; Schwabe and Wolf, 2009), we hypothesized that stress would impair the use of prior knowledge during learning (ie, schema-based learning). With respect to the role of glucocorticoids and noradrenaline, it was tempting to predict that both systems might act synergistically to disrupt schema-based learning.

\section{MATERIALS AND METHODS}

\section{Experiment I: Impact of Stress on Schema-Based Learning}

Participants. Ninety-six healthy individuals (48 female; age $(\mathrm{M} \pm \mathrm{SEM}): 25.24 \pm 0.36$ years $)$, without life-time history of any neurological or mental disorders, current pharmacological treatment or medication intake within the four weeks prior to participation, tobacco- or drug-use, over- or underweight, or intake of hormonal contraceptives in females participated in this experiment. Women were not invited for an appointment during their menses. Participants gave written informed consent before taking part in the study and received a compensation of $20 €$. The local ethics committee approved the study protocol. Five participants had to be excluded from the analyses because of sickness on experimental day 2 or inadequate knowledge of German, leaving a sample of 91 participants (45 females; Supplementary Material).

Experimental design and procedure. We used a two-day between-subjects design with the factors treatment (control $v s$ stress) and interval (immediate $v s$ delayed learning), resulting in four experimental groups to which participants were randomly assigned ( $n=22-24$ /group). All testing took place between 1300 and 1800 hours. On day 1, participants learned a hierarchy of six galaxies. On day $2, \sim 24 \mathrm{~h}$ after day 1, participants underwent a stress or control manipulation and learned both a novel hierarchy of galaxies and a hierarchy containing both elements of the hierarchy learned on day 1 and new elements (related hierarchy) either immediately or $25 \mathrm{~min}$ after the stress or control manipulation.
Stress manipulation. Participants in the stress condition were exposed to the Socially Evaluated Cold Pressor Test (SECPT), as described in detail elsewhere (Schwabe et al, 2008). In short, participants were instructed to submerge their left hand into ice water $\left(0-2^{\circ} \mathrm{C}\right)$ for $3 \mathrm{~min}$. A cold and nonreinforcing experimenter took notes to prompt a feeling of being evaluated. Furthermore, participants were videotaped for pretended analysis of facial expression. In the control condition, participants were instructed to immerse their hand into warm water $\left(35-37^{\circ} \mathrm{C}\right)$ for $3 \mathrm{~min}$. There was no camera nor were they being evaluated by the experimenter.

To assess the successful stress induction by the SECPT, we took subjective and physiological measurements repeatedly across the experiment. Immediately after the SECPT or control manipulation participants rated on a scale from 0 ('not at all') to 100 ('very much') how stressful, difficult, painful, and unpleasant they experienced the treatment. Blood pressure was measured using a Dinamap system (Critikon, FL, USA) at the beginning of the experiment, during the SECPT/control manipulation, immediately after the SECPT/control manipulation, before and after the task. For salivary cortisol measurements, saliva samples were collected using Salivette collection devices (Sarstedt, Germany) at baseline, immediately after the SECPT/control manipulation, as well as 10 and 30 min after the treatment; in the delayed learning groups also 40 and $60 \mathrm{~min}$ after the treatment. Saliva samples were stored at $-18^{\circ} \mathrm{C}$. Free concentrations of cortisol were analyzed subsequently by means of a luminescence assay (IBL, Germany; intra- and inter-assay coefficients of variance below 13 percent).

Learning task. To assess schema-based learning processes, we used a modified version of a task previously introduced by Kumaran and colleagues (Kumaran, 2013; Kumaran et al, 2012) that consisted of two stages: a schema-acquisition phase (phase I) and a schema-based learning phase (phase II).

Schema-acquisition phase (phase I). Phase I testing was completed on day 1 . In this phase, six galaxies were presented with a pre-determined age-hierarchy $(\mathrm{A}>\mathrm{B}>\mathrm{C}>\mathrm{D}>\mathrm{E}>\mathrm{F}$, with A being the 'oldest' galaxy; Figure 1). Participants were presented with three different types of trials: baseline-, learning- and inference trials. On each trial, participants saw two different galaxies next to each other. During baseline trials, a cross was presented below one of the images and participants were required to indicate under which image the cross was located by button press within $2 \mathrm{~s}$. A blank screen was shown for $0.5 \mathrm{~s}$, followed by feedback (green frame around the correct image) presented for $2 \mathrm{~s}$. Baseline trials served as control trials, probing for attention across the task. During learning trials, participants were presented with two neighboring galaxies (eg, A-B) and asked to indicate which of the galaxies was older. Feedback and timings were exactly the same as during baseline trials. During inference trials, participants were asked to indicate which of two non-neighboring galaxies, separated by an age distance of two (ie, short: eg, A-D), three or four (ie, long: eg, A-E, A-F), was older. Furthermore, participants did not receive corrective feedback in inference trials but had to indicate their certainty on a 4-item scale from 'I'm guessing' 

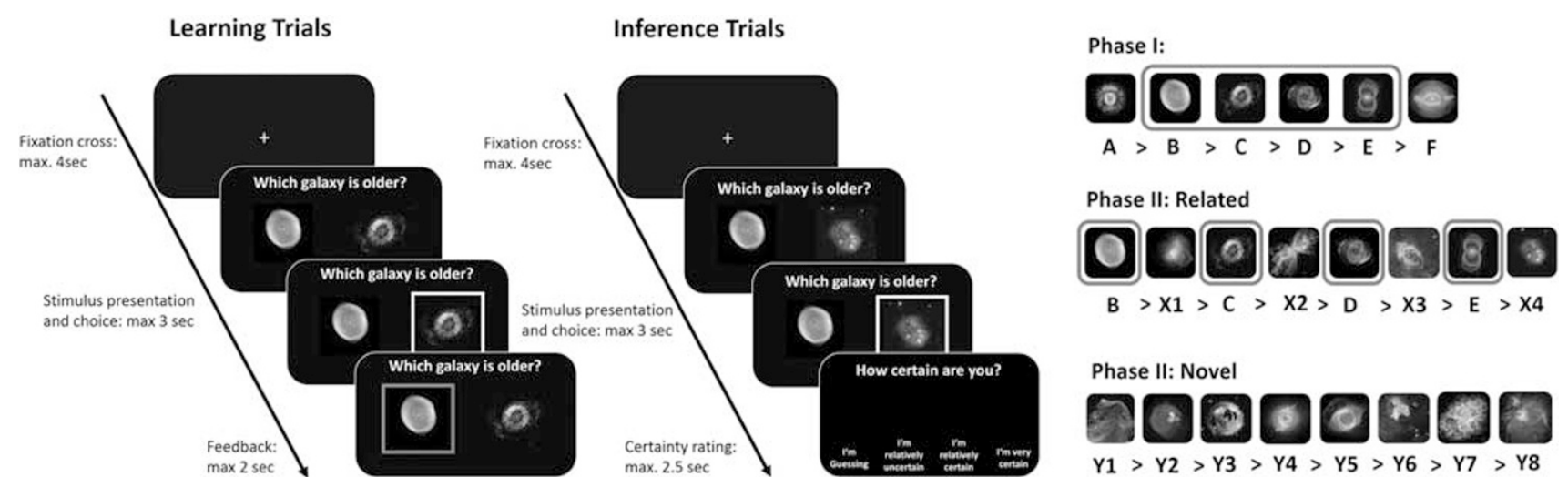

Phase II: Novel

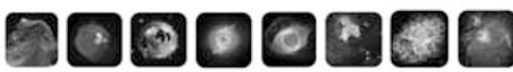

$\mathrm{Y} 1>\mathrm{Y} 2>\mathrm{Y} 3>\mathrm{Y} 4>\mathrm{Y} 5>\mathrm{Y} 6>\mathrm{Y} 7>\mathrm{Y} 8$

Figure I Learning task. In phase I, participants learned an age-hierarchy of six galaxies based on trial-by-trial feedback (learning trials, only neighboring galaxies were presented). In inference trials, participants were required to decide which of two non-neighboring items was older, without receiving feedback about the correct answer. In phase II, $24 \mathrm{~h}$ after phase I, participants were presented with two hierarchies comprising 8 galaxies each. One of the hierarchies (related) contained four galaxies already learned in phase I, and four completely new galaxies. The other hierarchy (novel) contained only new galaxies. Participants learned the positions of the new items again through trial and error in learning trials. In inference trials, the ability to put new items into the already existing hierarchy was tested, as only non-neighboring items were presented. Schema-based memory is reflected in better learning performance for the related compared with the novel hierarchy in phase II.

to 'I'm very certain' within $2 \mathrm{~s}$. After each trial, participants saw a fixation cross for a duration of 2-4 s.

During phase I, which took about $35 \mathrm{~min}$, participants completed 10 blocks, each comprising 4 baseline-, 10 training and 10 test trials (ie, 240 trials in total). The galaxies presented in each block were pre-determined, the order of galaxies presented was randomized.

Schema-based learning (phase II). Phase II testing was completed on day $2, \sim 24 \mathrm{~h}$ after day 1 and either immediately or $25 \mathrm{~min}$ after the SECPT or control procedure. In phase II, participants learned two hierarchies, each comprising 8 galaxies. One of the hierarchies contained 4 galaxies from the hierarchy learned during phase I and 4 completely new items $(\mathrm{B}>\mathrm{X} 1>\mathrm{C}>\mathrm{X} 2>\mathrm{D}>\mathrm{X} 3>\mathrm{E}>\mathrm{X} 4$-related hierarchy; see Figure 1). Participants were explicitly instructed that the hierarchy they learned in phase I was still true in phase II. The second hierarchy contained only new galaxies $(\mathrm{Y} 1>\mathrm{Y} 2>\mathrm{Y} 3>\mathrm{Y} 4>\mathrm{Y} 5>\mathrm{Y} 6>\mathrm{Y} 7>\mathrm{Y} 8$-novel, Figure 1). Participants again completed baseline, learning, and inference trials for both hierarchies in the same way as in phase I. Importantly for the related hierarchy, learning trials always consisted of one galaxy from phase I and one new item. Inference trials, however, involved only galaxies that had not been shown on day 1 .

For each hierarchy, related and novel, participants completed 6 blocks, comprising 2 baseline, 7 learning and 6 inference trials (180 trials in total). Related and novel blocks alternated and a change between them was not specifically indicated. Phase II testing lasted $\sim 23 \mathrm{~min}$. The galaxy images used in novel and related hierarchies as well as the hierarchy participants began with were counterbalanced.

\section{Experiment II: Impact of Cortisol and Noradrenaline on Schema-Based Learning}

Participants and experimental design. Ninety-six healthy participants were recruited for this experiment (48 female; age $(\mathrm{M} \pm \mathrm{SEM}): 24.78 \pm 0.39$ years, body-mass-index $(\mathrm{M} \pm \mathrm{SEM})$ : $\left.22.79 \pm 0.2 \mathrm{~kg} / \mathrm{m}^{2}\right)$. Exclusion criteria for participation were identical to experiment I. In addition, participants were screened for hydrocortisone intolerance, cardiovascular disorders, including low and high blood pressure and diabetes as well as related disorders. Participants gave written informed consent before taking part in the study and received a compensation of $35 €$ for participation. The ethics committee of the Hamburg Medical Association approved the study protocol. Six participants had to be excluded from the analyses due to insufficient schema acquisition on day 1 (learning trial performance below $40 \%$ and/or combined performance of learning and inference trials below chance level), most likely reflecting difficulties in understanding the task instructions, leaving a sample of 90 participants (44 females, Supplementary Material).

Experimental design and procedure. We used a two-day, double-blind, fully-crossed, placebo-controlled, betweensubjects design with the factors noradrenaline (placebo vs yohimbine) and cortisol (placebo vs hydrocortisone), resulting in four experimental groups to which participants were randomly assigned ( $n=22$ or 23 per group). All testing took place between 1300 and 1900 hours. On day 1, participants completed the first phase of the learning task (ie, schema acquisition). On day $2, \sim 24 \mathrm{~h}$ after day 1 , participants were administered the drugs 45 min before they completed phase II of the task (ie, schema-based learning).

Pharmacological manipulation. Participants received orally either a placebo (12 male, 10 female), $20 \mathrm{mg}$ hydrocortisone (12 male, 11 female), $20 \mathrm{mg}$ yohimbine (10 male, 12 female), a $\alpha 2$-adrenoceptor-antagonist leading to increased noradrenergic stimulation, or both drugs (12 male, 11 female). Timing and dosages of drug administration were chosen in accordance with previous studies (Buchanan and Lovallo, 2001; Schwabe et al, 2010a). To validate the action of the drugs, blood pressure and salivary cortisol were measured before medication intake, $45 \mathrm{~min}$ after medication intake and after phase II of the learning task (about $70 \mathrm{~min}$ after drug intake). 

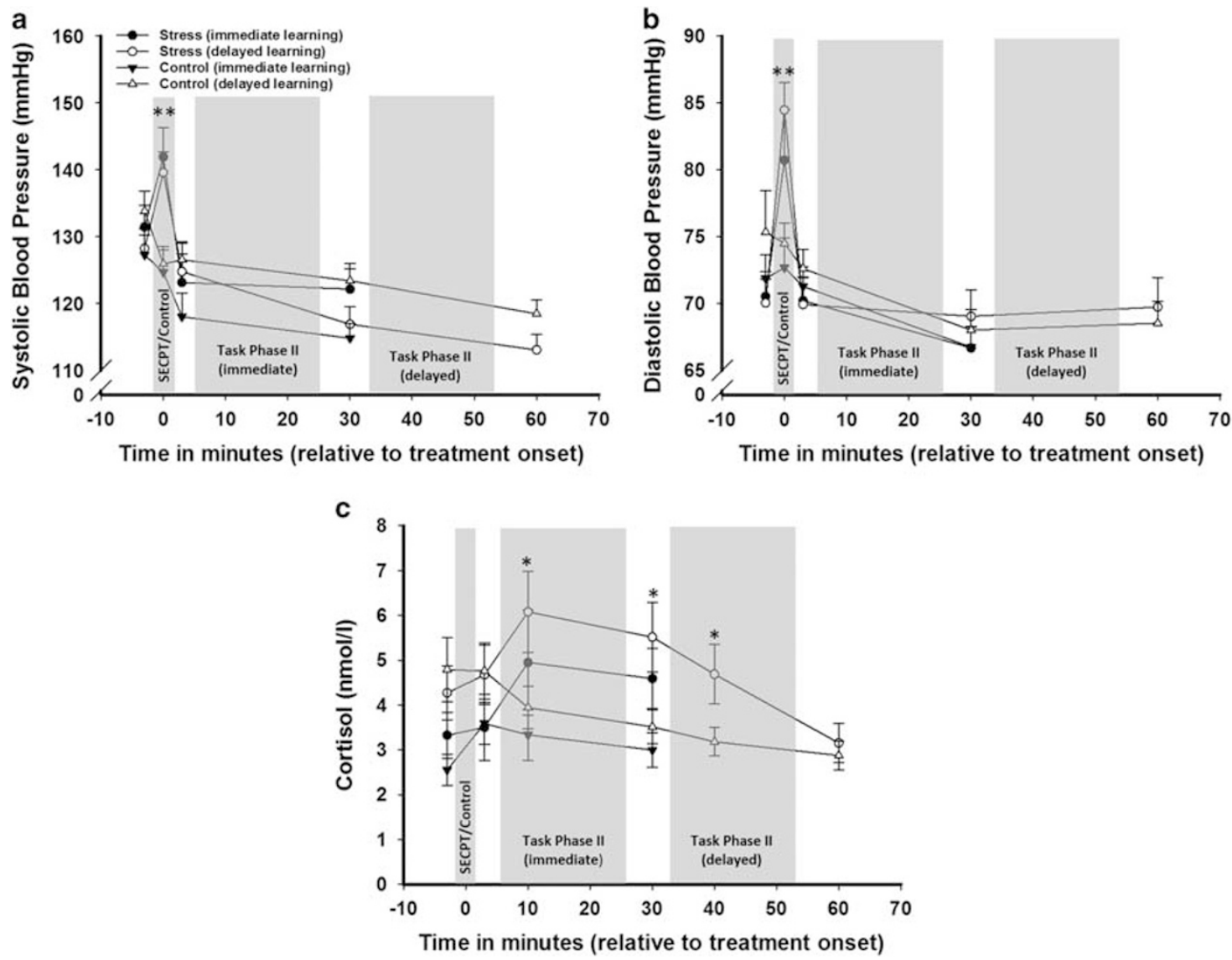

Figure 2 Successful stress induction in experiment I. The exposure to the Socially Evaluated Cold Pressor Test (SECPT) led to a significant increase in (a) systolic blood pressure and (b) diastolic blood pressure, whereas the exposure to the control manipulation did not. (c) Salivary cortisol levels were significantly increased in participants undergoing the SECPT, compared with participants that were subjected to the control manipulation. Data represent means \pm SEM *** $p<0.00$ I, ${ }^{*} p<0.05$.

Learning task. The learning task was identical as in experiment I, with two exceptions. First, the number of blocks of phase I was reduced to 15 blocks comprising 180 trials because participants already showed robust learning after 180 trials in experiment I. Second, we presented an explicit hierarchy recall test for the hierarchy learned on day 1 about $45 \mathrm{~min}$ after pill intake on day 2 (before phase II started), in order to rule out that potential drug effects are due to a simple memory retrieval deficit.

An overview of the statistical analyses is given in the Supplementary Material.

\section{RESULTS}

\section{Experiment I: Stress Impairs Schema-Based Learning}

Significant increases in subjective stress level, blood pressure and cortisol confirmed the successful stress induction by the SECPT. There were no significant differences between the immediate and delayed groups (Figure 2 and Supplementary Results).

Phase I: Successful schema acquisition. Participants showed a significant increase in performance in both learning and inference trials (both $p<0.001, \eta_{\mathrm{p}}=0.279$; Supplementary Figure S1), with a performance of 81 percent in the learning trials and 77 percent correct responses in the inference trials the end of the acquisition phase, indicating that participants acquired a stable schema. Baseline trial performance was close to perfect (on average 92 percent correct, Supplementary Table S5). Most importantly, experimental groups did not differ in their performance on day 1 , ie, they acquired the schema equally well (all effects including the factor experimental group: all $\mathrm{F}<2.02$, all $p>0.12$ ).

Phase II: Schema-based learning under stress. Overall, participants showed increasing performance independent of hierarchy types $\left(\mathrm{F}_{(3.97,344.98)}=30.180, p<0.001, \eta_{\mathrm{p}}=0.258\right.$, Supplementary Figure S2A). Performance tended to be better in learning trials for the related compared to the novel hierarchy $\left(\mathrm{F}_{(1,87)}=3.519, p=0.064, \eta_{\mathrm{p}}=0.039\right)$, reflecting the expected schema-effect. Most importantly, however, stress affected the extent to which prior knowledge facilitated performance. Participants in the control groups performed significantly better in related compared with novel trials (71 vs 64 percent in the last block; $\mathrm{F}_{(1,43)}=7.976, p=0.007$, $\left.\eta_{\mathrm{p}}=0.156\right)$ which demonstrates a schema-based memory effect. Stressed participants, however, did not perform better in related compared to novel trials (62 and 60 percent, respectively; $\mathrm{F}_{(1,44)}=0.003, p=0.959, \eta_{\mathrm{p}}<0.001 ;$ marginal hierarchy type $\times$ treatment interaction: $\left.F_{(1,} 87\right)=3.806$, 
$p=0.054, \eta_{\mathrm{p}}=0.042$, Figure 3 ). Importantly, this effect of stress was comparable in the immediate learning and delayed learning groups (hierarchy type $\times$ treatment $\times$ interval and hierarchy type $\times$ block $\times$ treatment $\times$ interval interactions, both $\mathrm{F} \leqslant 1.192$, both $p \geqslant 0.313$ ). Reaction times did not differ between groups (all $\mathrm{F} \leqslant 1.569$, all $\mathrm{p} \geqslant 0.184$ ). In baseline trials, participants reached an average performance of 98 percent. Because inference trials are less dependent on the medial PFC (Heckers et al, 2004) and reflect cognitive processes that are beyond the scope of the present experiment, data from these trials are presented in the Supplementary Material.

\section{Experiment II: Hydrocortisone, but not Yohimbine, Disrupts Schema-Based Learning}

As expected, hydrocortisone led to an increase in salivary cortisol but not to a change in blood pressure. Conversely, yohimbine increased blood pressure but not salivary cortisol (Figure 4 and Supplementary Results).

Phase I: Successful schema acquisition. Participants showed a significant increase in performance in both learning and inference trials across phase I of the task (both $\mathrm{F}>16.65$, both $p<0.001$, both $\eta_{\mathrm{p}}>0.16$, Supplementary Figure S4). At the end of the acquisition phase, participants were correct on about 80 percent of the learning and inference trials, indicating that participants acquired a robust schema. Performance in baseline trials reached 92 percent on average (Supplementary Table S7). Notably, the experimental groups did not differ in schema acquisition (all $\mathrm{F}<1.28$, all $p>0.14)$.

Phase II: Schema-based learning after hydrocortisone and yohimbine intake. The explicit recall of the hierarchy learned on day 1 remained unaffected by yohimbine and hydrocortisone (all $\mathrm{F} \leqslant 2.720$, all $p \geqslant 0.103$ ), thus showing that the drugs did not affect the simple retrieval of the hierarchy learned on day 1. Furthermore, participants showed almost perfect performance on baseline trials and reached 98 percent on average.

Performance in the learning trials increased over time $\left(\mathrm{F}_{(5,430)}=39.929, \quad p<.001, \eta_{\mathrm{p}}=0.317\right)$ and was significantly better for related compared to novel trials, demonstrating the expected schema-effect $\left(\mathrm{F}_{(1,86)}=18.459\right.$, $p<0.001, \eta_{p}=0.177$, Supplementary Figure S5A). Most importantly, however, we obtained a significant interaction of hierarchy type (ie, novel $v s$ related) and cortisol $\left(\mathrm{F}_{(1,86)}=8.335, p=0.005, \eta_{\mathrm{p}}=0.088\right.$, Figure 5), indicating that hydrocortisone modulated participants' ability to utilize previous knowledge during learning. Specifically, both participants in the placebo group and those in the yohimbine only group showed significantly better performance in related than in novel trials (both $t \leqslant 2.616$, both $p \leqslant 0.016$ ). Participants that had received hydrocortisone, however, whether alone or in combination with yohimbine, did not benefit from the prior knowledge and performed comparably in novel and related trials (both $t \leqslant 0.673$, both $p \geqslant 0.465$ ). While hydrocortisone modulated schema-based learning, there was no main effect of yohimbine and no hydrocortisone $\times$ yohimbine interaction on schema-based learning (all $\mathrm{F} \leqslant 1.595$, all $p \geqslant 0.160$ ). Reaction times did not differ

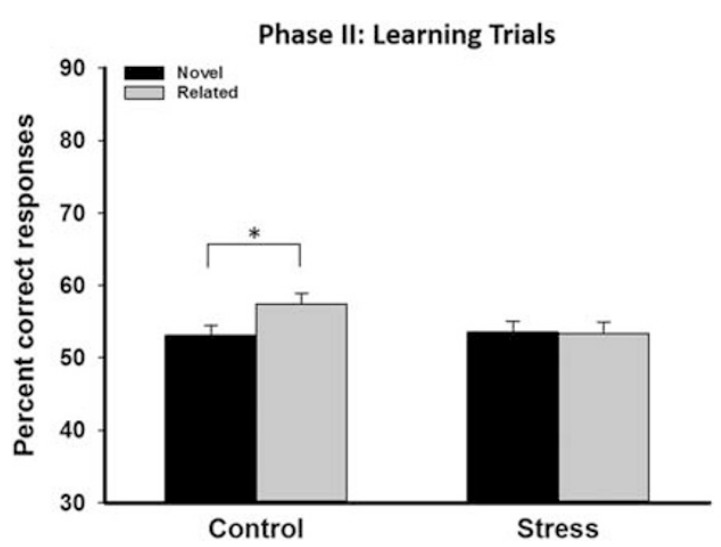

Figure 3 Performance in leaming trials in phase II experiment I. Learning trial performance was improved for the related compared to novel hierarchy in control participants, indicating the successful utilization of a pre-existing schema. Stressed participants, however, did not perform better in related vs novel trials, indicating a reduced ability to utilize prior knowledge during learning, ie, impaired schema-based memory. Data represent means \pm SEM. $* p<0.05$.

between groups $(\mathrm{F} \leqslant 1.221, p \geqslant 0.301)$. The data from the inference trials are presented and discussed in the Supplementary Material.

\section{DISCUSSION}

Prior knowledge, a 'schema', is known to facilitate the encoding, consolidation, and retrieval of schema-related information (Alba and Hasher, 1983; Ghosh and Gilboa, 2014a; Morris, 2006; Tse et al, 2007; van Kesteren et al, 2012). In the present experiments, we examined the impact of stress and major stress mediators, ie, cortisol and noradrenaline, on schema-based learning. Our findings show that stress interferes with the ability to utilize a pre-existing schema to aid learning of new information. A similar impairment of schema-based learning was observed after hydrocortisone administration, but not after administration of the $\alpha 2$ adrenoceptor antagonist yohimbine, suggesting that glucocorticoid, but not noradrenergic activity is sufficient to hinder the use of prior knowledge during learning.

Converging lines of evidence from rodent and human neuroimaging studies identified the medial PFC as a key locus for schema-based memory processes (Tse et al, 2007; Tse et al, 2011; van Kesteren et al, 2013). The PFC expresses receptors for glucocorticoids at a particularly high density (McEwen et al, 1986) and stressful events may impair prefrontal functions (Arnsten, 2009; Izquierdo et al, 2006; Schwabe and Wolf, 2009). Accordingly, it appears reasonable to assume that the observed stress-induced deficit in schemabased learning was due to an impairing effect on the medial PFC, reducing its ability to detect the congruency of new information with an existing schema. Interestingly, the impairing effect of stress on schema-based learning was observed both when learning took place immediately after the stressful event and when it took place about 25 min later. Given that noradrenaline levels were most likely still increased in the immediate learning condition but not in the delayed learning condition, whereas cortisol levels were already rising during learning in the immediate learning condition and reached peak levels shortly before or during 

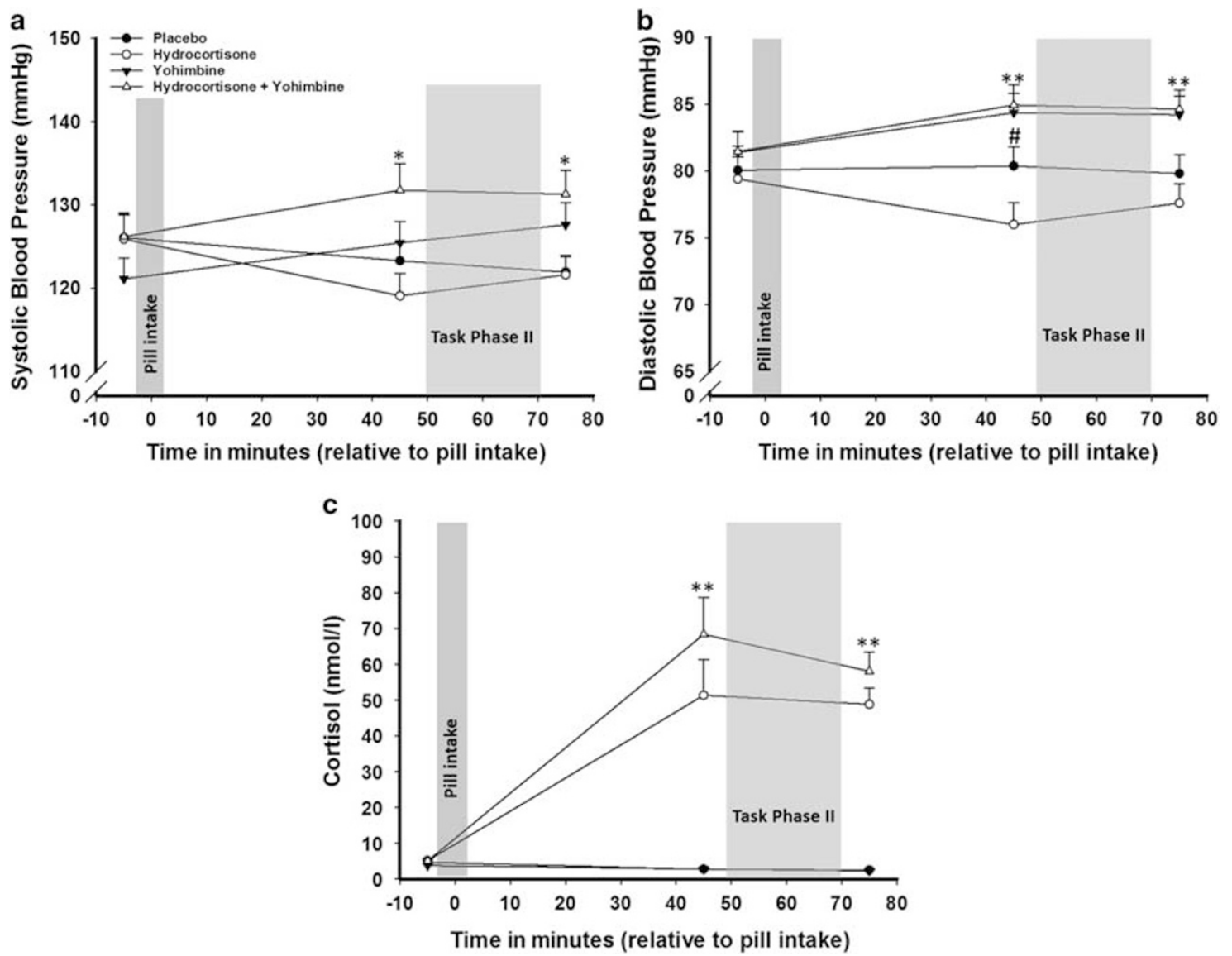

Figure 4 Effectiveness of the pharmacological manipulation in experiment II. Participants that received solely yohimbine or yohimbine in combination with hydrocortisone showed a significant increase in (a) systolic and (b) diastolic blood pressure, compared to participants that received a placebo or hydrocortisone only. 45 min after pill intake the placebo group showed a strong trend for a higher diastolic blood pressure, compared to the hydrocortisone only group. (c) Salivary cortisol levels were significantly increased in participants that received hydrocortisone only or in combination with yohimbine, compared with participants that received yohimbine only or a placebo. Data represent means \pm SEM $* * p<0.00 I, * p<0.05,{ }^{\#} p<0.10$.

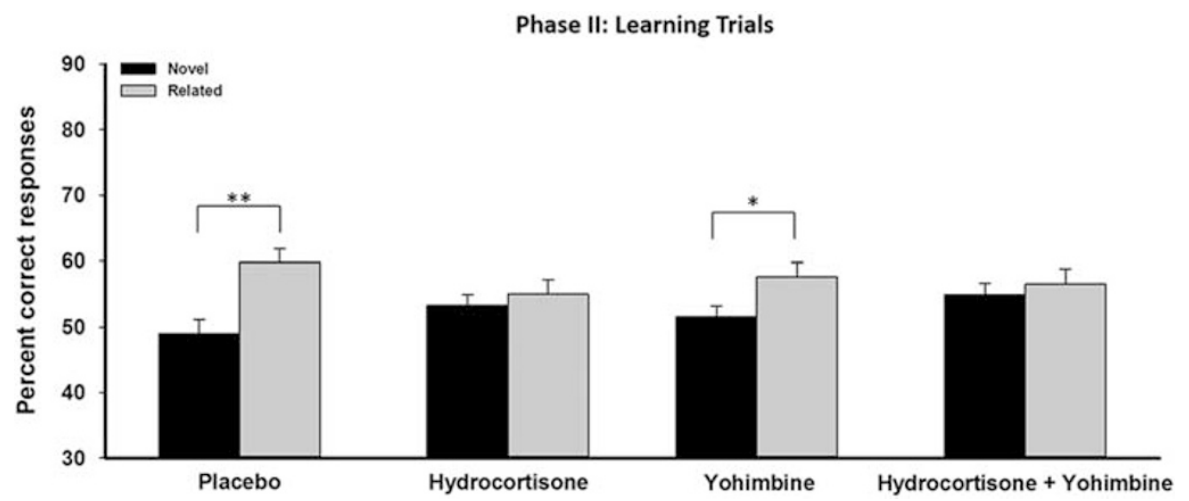

Figure 5 Performance in learning trials in phase II in experiment II. Placebo and yohimbine only groups showed a significant schema-based memory effect, indicated by better performance for related compared to novel hierarchies. This schema-based memory effect was absent in participants that received hydrocortisone alone or in combination. Data represent means \pm SEM. * $p<0.05$.

learning in the delayed condition, this finding suggests that the stress-induced deficit in schema-based learning was rather owing to the action of glucocorticoids than to noradrenaline.

The idea that glucocorticoids have a major role in the modulation of schema-based learning is supported by our pharmacological experiment. This experiment showed that a moderate dose of hydrocortisone led to an impairment in the use of prior knowledge that resembled the deficit observed after stress, suggesting that a rise in glucocorticoid levels is sufficient to impair schema-based learning processes. Previous studies indicated that prefrontal structures that are critically involved in schema-based learning are a major target of glucocorticoids (Liu and Aghajanian, 2008; Meaney et al, 1985). Thus, glucocorticoid-induced changes in prefrontal areas are a likely mechanism underlying the 
impairment of schema-based learning after hydrocortisone intake. The classical mode of action of glucocorticoids involves intracellular glucocorticoid and mineralocorticoid receptors (GR and $M R$, respectively) that mediate relatively slow genomic changes by transactivation and transrepression of responsive genes (De Kloet et al, 1986; Joels et al, 2012). More recent research, however, indicates that there are also membrane-bound MR and GR that initiate rapid, nongenomic changes in neural excitability, cognition and behavior (Barsegyan et al, 2010; Joels et al, 2012; Roozendaal et al, 2010). Given the time-scale of the observed glucocorticoid effects on schema-based memory, we argue that these effects were most likely mediated by membranebound receptors. Future studies using selective MR and GR antagonists, possibly in combination with glucocorticoids conjugated to a membrane-impermeable bovine serum albumin molecule, are required to elucidate the specific role of these receptor types in the impact of glucocorticoids on the use of prior knowledge during learning.

Although our data show that glucocorticoid elevations are sufficient to impair schema-based learning, no such effect was found after increased noradrenergic stimulation by yohimbine. Previous studies showed that noradrenaline may affect PFC-dependent cognitive functions (Ramos and Arnsten, 2007) and that noradrenaline may interact with glucocorticoids in influencing prefrontal functions (Barsegyan et al, 2010; Schwabe and Wolf, 2010b, 2012c). However, we also obtained no evidence for an interaction of glucocorticoids and noradrenaline, neither in our pharmacological experiment nor in the stress experiment, in which a more pronounced effect in the immediate compared to the delayed condition may have been indicative for such an interaction. One potential explanation for the absence of an effect of noradrenaline may be seen in the specific cognitive function tested. Schema-based memory is centered on the mPFC interacting with a large network of other regions (van Kesteren et al, 2012) and whether noradrenaline may affect schema-based memory processes has not been tested before. Moreover, while noradrenergic activation appears not to be sufficient for the modulation of schema-based memory, it may well be necessary for stress and glucocorticoids to exert their detrimental effects on the use of prior knowledge during learning. In the stress experiment, noradrenaline levels were initially raised in all stressed participants and the pill intake and task performance was most likely associated with a certain level of arousal in the pharmacological experiment. Future studies may combine a stress or glucocorticoid manipulation with $\beta$-adrenergic receptor blockade to examine whether noradrenaline is necessary in enabling the impairing effects of stress and glucocorticoids on schema-based memory.

Finally, it is important to note that the task we used here was rather difficult and, in line with previous findings (Kumaran, 2013), performance was far from perfect in this task, even after initial learning on day 1. Thus, one might question, whether participants did acquire a robust schema. However, the fact that, in controls, performance was significantly better in related compared with novel trials suggests that they could benefit from their prior knowledge. Moreover, inference trial performance depended significantly on the distance between the hierarchy elements (Supplementary Material), which strongly suggests that participants had indeed constructed an associative structure (ie, a schema).

To conclude, whereas stress effects on memory formation or retrieval are very well documented (Diamond et al, 2007; Joels et al, 2011; Roozendaal et al, 2009; Schwabe et al, 2012a), we examined here the integration of new information and prior knowledge, ie, schema-based memory. Our findings go beyond the known effects of stress on memory and show that stress reduces the ability to benefit from prior knowledge during learning. Importantly, the present effects of stress and major stress mediators could be separated from the classical effects on memory formation and memory retrieval. Specifically, both the encoding of the novel hierarchy and (in experiment 2) the recall of the hierarchy learned on day 1 remained unaffected by our experimental manipulation on day 2 (Supplementary Discussion), thus making simple stress or glucocorticoid effects on encoding or retrieval unlikely. Instead, we argue that stress and glucocorticoids affected primarily the schema-congruency detection by the medial PFC, that is thought to facilitate the integration of schema-related information into neocortical memory representations (van Kesteren et al, 2012). On the basis of our finding that the administration of glucocorticoids was sufficient to produce a deficit in the ability to use prior knowledge during learning that strongly resembled the effect observed after stress, it is tempting to speculate that glucocorticoids play a key role in the stress-induced impairment of schema-based memory. The present results may have important implications for educational settings, in which the ability to utilize prior knowledge during learning is essential and in which stressful events are very common.

\section{FUNDING AND DISCLOSURE}

The authors declare no conflict of interest.

\section{ACKNOWLEDGMENTS}

We gratefully acknowledge the assistance of Sonja Timmerman, Olivia Bendlin, Mewes Muhs, Michaela Christoph, Sandra Weber, and Irmak Bagirsakci during data collection. This study was supported by funding received from the German Research Foundation (DFG; grants SCHW 1357/5-3 and 1357/14-1).

\section{REFERENCES}

Alba JW, Hasher L (1983). Is memory schematic? Psychol Bull 93: 203-231.

Anderson R (1984). Some reflections on the acquisition of knowledge. Educ Res 13: 5-11.

Arnsten AF (2009). Stress signalling pathways that impair prefrontal cortex structure and function. Nat Rev Neurosci 10: 410-422.

Barlett FC (1932). Remembering: A study In experimental and social psychology. University Press: Cambridge.

Barsegyan A, Mackenzie SM, Kurose BD, McGaugh JL, Roozendaal B (2010). Glucocorticoids in the prefrontal cortex enhance memory consolidation and impair working memory by a common neural mechanism. Proc Natl Acad Sci USA 107: 16655-16660.

Buchanan TW, Lovallo WR (2001). Enhanced memory for emotional material following stress-level cortisol treatment in humans. Psychoneuroendocrinology 26: 307-317. 
De Kloet ER, Reul JMHM, De Ronde FSW, Bloemers M, Ratka A (1986). Function and plasticity of brain corticosteroid receptor systems: action of neuropeptides. J Steroid Biochem 25: 723-731.

Diamond DM, Campbell AM, Park CR, Halonen J, Zoladz PR (2007). The temporal dynamics model of emotional memory processing: a synthesis on the neurobiological basis of stressinduced amnesia, flashbulb and traumatic memories, and the Yerkes-Dodson law. Neural Plast 2007: 1-32.

Elzinga BM, Roelofs K (2005). Cortisol-induced impairments of working memory require acute sympathetic activation. Behav Neurosci 119: 98-103.

Ghosh VE, Gilboa A (2014a). What is a memory schema? A historical perspective on current neuroscience literature. Neuropsychologia 53: 104-114.

Ghosh VE, Moscovitch M, Melo Colella B, Gilboa A (2014b). Schema representation in patients with ventromedial PFC lesions. J Neurosci 34: 12057-12070.

Heckers S, Zalesak M, Weiss AP, Ditman T, Titone D (2004). Hippocampal activation during transitive inference in humans. Hippocampus 14: 153-162.

Izquierdo A, Wellman CL, Holmes A (2006). Brief uncontrollable stress causes dendritic retraction in infralimbic cortex and resistance to fear extinction in mice. J Neurosci 26: 5733-5738.

Joels M, Fernandez G, Roozendaal B (2011). Stress and emotional memory: a matter of timing. Trends Cogn Sci 15: 280-288.

Joels M, Sarabdjitsingh RA, Karst H (2012). Unraveling the time domains of corticosteroid hormone influences on brain activity: rapid, slow, and chronic modes. Pharmacol Rev 64: 901-938.

Kumaran D (2013). Schema-driven facilitation of new hierarchy learning in the transitive inference paradigm. Learn Mem 20: 388-394.

Kumaran D, Melo HL, Duzel E (2012). The emergence and representation of knowledge about social and nonsocial hierarchies. Neuron 76: 653-666.

Liu RJ, Aghajanian GK (2008). Stress blunts serotonin- and hypocretin-evoked EPSCs in prefrontal cortex: role of corticosterone-mediated apical dendritic atrophy. Proc Natl Acad Sci USA 105: 359-364.

McEwen BS, De Kloet ER, Rostene W (1986). Adrenal steroid receptors and actions in the nervous system. Physiol Rev 66: $1121-1188$.

Meaney MJ, Aitken DH, Bodonoff SR, Iny LJ, Tatarewicz JE, Sapolsky RM (1985). Early postnatal handling alters glucocorticoid receptor concentrations in selected brain regions. Behav Neurosci 99: 765-770.

Moench KM, Maroun M, Kavushansky A, Wellman C (2016). Alterations in neuronal morphology in infralimbic cortex predict resistance to fear extinction following acute stress. Neurobiol Stress 3: 23-33.

Morris RG (2006). Elements of a neurobiological theory of hippocampal function: The role of synaptic plasticity, synaptic tagging and schemas. Eur J Neurosci 23: 2829-2846.
Ramos BP, Arnsten AF (2007). Adrenergic Pharmacology and cognition: Focus on the prefrontal cortex. Pharmacol Therapeut 113: $523-546$.

Roozendaal B, Hernandez A, Cabrera SM, Hagewoud R, Malvaez M, Stefanko DP et al (2010). Membrane-associated glucocorticoid activity is necessary for modulation of long-term memory via chromatin modification. J Neurosci 30: 5037-5046.

Roozendaal B, McEwen BS, Chattarji S (2009). Stress, memory and the amygdala. Nat Rev Neurosci 10: 423-433.

Schwabe L, Haddad L, Schachinger H (2008). HPA axis activation by a socially evaluated cold-pressor test. Psychoneuroendocrinology 33: 890-895.

Schwabe L, Hoffken O, Tegenthoff M, Wolf OT (2011). Preventing the stress-induced shift from goal-directed to habit action with a beta-adrenergic antagonist. J Neurosci 31: 17317-17325.

Schwabe L, Joels M, Roozendaal B, Wolf OT, Oitzl MS (2012a). Stress effects on memory: an update and integration. Neurosci Biobehav Rev 36: 1740-1749.

Schwabe L, Tegenthoff M, Hoffken O, Wolf OT (2010a). Concurrent glucocorticoid and noradrenergic activity shifts instrumental behavior from goal-directed to habitual control. J Neurosci 30: 8190-8196.

Schwabe L, Tegenthoff M, Hoffken O, Wolf OT (2012b). Simultaneous glucocorticoid and noradrenergic activity disrupts the neural basis of goal-directed action in the human brain. J Neurosci 32: 10146-10155.

Schwabe L, Wolf OT (2009). Stress prompts habit behavior in humans. J Neurosci 29: 7191-7198.

Schwabe L, Wolf OT (2010b). Socially evaluated cold pressor stress after instrumental learning favors habits over goal-directed action. Psychoneuroendocrinology 35: 977-986.

Schwabe L, Wolf OT (2012c). Stress modulates the engagement of multiple memory systems in classification learning. J Neurosci 32: 11042-11049.

Tse D, Langston RF, Kakeyama M, Bethus I, Spooner PA, Wood ER et al (2007). Schemas and memory consolidation. Science 316: 76-82.

Tse D, Takeuchi T, Kakeyama M, Kajii Y, Okuno H, Tohyama C et al (2011). Schema-dependent gene activation and memory encoding in neocortex. Science 333: 891-895.

Valentin VV, Dickinson A, O'Doherty JP (2007). Determining the neural substrates of goal-directed learning in the human brain. J Neurosci 27: 4019-4026.

van Kesteren MT, Beul SF, Takashima A, Henson RN, Ruiter DJ, Fernandez G (2013). Differential roles for medial prefrontal and medial temporal cortices in schema-dependent encoding: from congruent to incongruent. Neuropsychologia 51: 2352-2359.

van Kesteren MT, Rijpkema M, Ruiter DJ, Fernandez G (2010). Retrieval of associative information congruent with prior knowledge is related to increased medial prefrontal activity and connectivity. J Neurosci 30: 15888-15894.

van Kesteren MT, Ruiter DJ, Fernandez G, Henson RN (2012). How schema and novelty augment memory formation. Trends Neurosci 35: 211-219.

Supplementary Information accompanies the paper on the Neuropsychopharmacology website (http://www.nature.com/npp) 